\title{
Improving Traffic in Urban Environments applying the Wardrop Equilibrium
}

\author{
Lara Codecà, Raphaël Frank, Thomas Engel \\ Interdisciplinary Centre for Security, Reliability and Trust \\ University of Luxembourg, 2721, Luxembourg \\ lara.codeca@uni.lu,raphael.frank@uni.lu,thomas.engel@uni.lu
}

\begin{abstract}
Over the last few years vehicular traffic density has continuously increased and it is likely that the traffic demand will further increase in the future. In many metropolitan areas the road infrastructure is no longer able to handle the peak traffic demand and the existing road network cannot be easily extended. The best way to improve the traffic situation is to optimise the resources available in the transportation network and to coordinate the traffic demand. The approach that we propose in this paper makes use of a collaborative traffic coordination protocol which collects real-time Floating Car Data (FCD) directly from participating vehicles and suggests dynamic routes in order to minimize travel delay. Information such as speed, position and direction is sent to one or more Traffic Coordination Points (TCPs) where it is aggregated to obtain a global picture of the traffic conditions in real-time. Based on this data, we continuously compute the route that minimizes the travel time to a given destination by applying Wardrop's first principle of equilibrium. Our results show that, by coordinating the vehicles, we are able to better distribute the overall traffic demand throughout the transportation network, reducing the average travel times and accommodating more vehicles.
\end{abstract}

Keywords-Intelligent Transportation Systems, Urban Traffic, Traffic Flow Optimization, Wardrop Equilibrium

\section{INTRODUCTION}

Traffic congestion is a real issue that most of the people living in any city have to face every day. Traffic density is increasing continually and, in many metropolitan areas, the road network has reached its limits and cannot easily be extended to meet the growing traffic demand. It is clear that public transportation services will play a major role in enabling mobility of commuters and workers. However, with the continuous growth of economic activities, the demand for individual mobility will likewise increase and thus must be considered.

The urban environment is characterised by short road segments and different types of intersections (e.g. right-before-left priority and traffic lights). Urban traffic comprises of different types of vehicles, pedestrians and unexpected obstacles. In recent decades, many models have been developed to describe and analyse vehicular mobility [1], [2], [3] and [4]. Most of these works have been proposed by civil engineers, mathematicians and physicists.

In order to match the growing traffic demand one possibility is to increase the capacity of the transportation network. This can be done by physically increasing the number of roads and their size; another option is to rearrange the topology of the network; or to optimise the usage of the resources already available. This last option can be achieved by collaborative traffic management systems that are able to orchestrate the routes of vehicles moving in metropolitan areas to reduce congestions.

The solution proposed in this paper is based on the usage of real-time Floating Car Data (FCD) which is aggregated to obtain an accurate and reliable overview of the traffic situation. The traditional traffic management systems are mainly composed of inductive loops, traffic cameras and roadside units deployed in the urban area. Such equipments have a high initial and maintenance costs and are prone to errors. Because the number of On-Board Units (OBU) (e.g. navigation systems and smartphones) has significantly increased over the last few years, this data that can now be retrieved on a large scale enabling the research community to explore other, more dynamic, approaches to improve traffic conditions. Several papers have recently proposed methods for efficiently sensing traffic-relevant information, among them [5], [6], [7], [8] and [9].

In this paper, the optimisation of the available resources is based on the concept of dynamically modifying the routes of the vehicles to better distribute the traffic demand all over the road network. To do this, the system continuously suggests to each vehicle the best route in terms of travel time to reach its destination, considering the actual traffic situation. This can be modelled as a flow optimisation problem [10], [11] and [12]. A different approach to finding alternative routes for the vehicles is to use the first Wardrop principle [13]. This principle is known as the user-optimal equilibrium and provides the best solution for each individual user.

We evaluated our proposal through simulation. The road topology for all the tested scenarios is a 10x10 Manhattan grid and the vehicles in the simulation are parametrised according to the Krauss car-following model [14]. The experiments were structured to investigate the performance of the proposed system in respect to the traffic density, the monitoring interval and the participation rate of vehicles. Another aspect that we investigated is the comparative traffic distribution in coordinated and uncoordinated traffic scenarios.

Our results show that by applying Wardrop's first principle of equilibrium we are able to substantially increase the amount of traffic served, improve the overall traffic fluidity and reduce the delays experienced by the vehicles.

The rest of the paper is organised as follows. Section II 
describes the urban environment and its complexity. Section III provides details of the communication protocol and the rerouting algorithm. Section IV describes the simulation environment and presents the results. Section V gives an overview of the related works and in Section VI we draw a conclusion and provide directions for future work.

\section{URBAN TRAFFIC DYNAMICS}

In order to mitigate traffic congestion and to optimise traffic flow it is necessary to define a model able to describe traffic dynamics. Over recent decades several models have been proposed. For example, [1] describes a multi-class traffic stream, based on the Lighthill-Whitham-Richards (LWR) model, which is able to define different drivers behaviours. Another model is the Krauss car-following model [14] and a simplification of it is presented in [2]. Another possibility is proposed in [15], and is based on a cellular automaton model. On motorways and long roads, a macroscopic traffic model based on traffic flow, traffic density and velocity forms the basis of the Macroscopic Fundamental Diagram [15]. This diagram can be used to predict the capacity of a road infrastructure and the traffic behaviour in relation to different inflow regulations and speed limits. Works such as [3] and [4] follow a similar direction but address the more complex urban road topology (e.g. the presence of regulated and unregulated intersections, various types of vehicles, pedestrians and unpredictable obstacles). The urban transportation infrastructure was designed decades ago to serve a defined amount of traffic. Nowadays the number of vehicles has increased significantly and the road network frequently reaches its capacity limit during rush hours. The naïve solution to this problem consists in modifying and expanding the infrastructure to match the increasing demand. This solution is not always feasible due to lack of available space or limited resources. Moreover, the Braess paradox [16] states that increasing the overall capacity of a network when the traffic is not coordinated can in some cases reduce the overall system performance. The approach that we propose is to coordinate the traffic by modifying the routes of the vehicles in the city to efficiently exploit the transportation infrastructure to better serve the traffic demand.

The traffic flow optimisation problem has been addressed with different methodologies and algorithms such as those presented in [10], [11] and [12]. In this paper we explore a different approach based on the selfish user-centred policy defined by Wardrop's first principal of equilibrium [13]. Wardrop equilibria are well-known solutions for network games based on transportation and telecommunication networks with congestion. The concept behind the first principle is that every player (vehicle in our context) will select a route that minimises the travel cost between two locations. We shall call $R_{o, d}$ the set of all the possible routes from the origin $o$ to the destination $d$ and the route chosen by the vehicle $i$ as route $i$.

$$
\text { route }_{o, d}^{i}=\min \left(R_{o, d}\right) .
$$

This principle is known as the user-optimal equilibrium and has become accepted [17] as a behavioural principle to describe the spreading of trips over alternate routes due to congested conditions. However, since users selfishly choose their routes
Fig. 1. Protocol Overview.

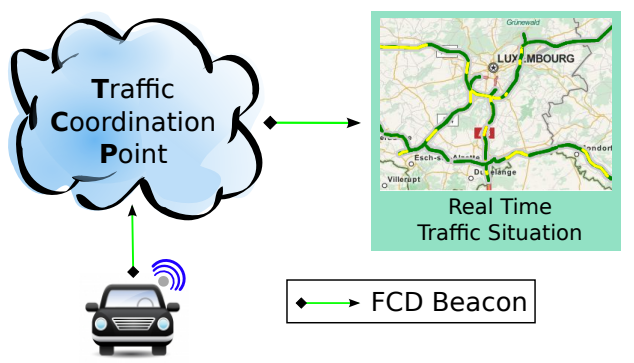

(a) Information Beaconing

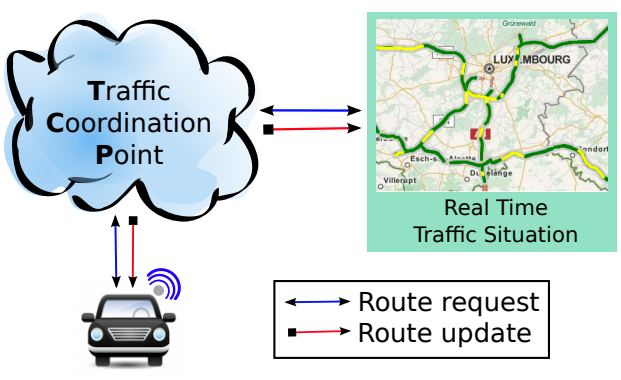

(b) Route Management

to minimise their costs, the solution is not necessarily systemoptimal. Wardrops second principle states that users minimise the total travel time in the system. Our aim is to find an efficient solution deployable in a city. The first principle being usercentred, implies an immediate benefit for each user and thus increases the chance of a greater initial acceptance.

\section{COMMUNICATION PROTOCOL}

The architecture of the system is based on a Vehicleto-Infrastructure (V2I) communication network in which the vehicles in the monitored area communicate with a local Traffic Coordination Point (TCP) using their On-Board Unit (OBU). The communication protocol is presented in Figure 1 and is divided in two parts: Information Beaconing and Route Management.

The first part is Information Beaconing 2(a). While the vehicles move through the road network, the OBU collects the traffic metrics (location, direction and speed) and sends them to the local TCP via a mobile data network (e.g. 3G/4G). The size of the beacon is small enough not to place any specific requirements on the connection in terms of bandwidth. The TCP aggregates these metrics continuously to update the traffic situation in real-time.

The second part is Route Management 2(b). The OBU sends the current location and desired destination to the local TCP, which uses the real-time traffic data to compute the fastest route in terms of travel time. The optimal route is sent as a reply to the OBU. If the optimal route changes due to the traffic dynamics, the OBU receives a route update.

The minimum delay route is computed using Dijkstra's algorithm [18] with dynamic edge costs in which the weight is the Estimated Travel Time (ETT). The ETT for a road segment is computed using its length, $l_{s}$, divided by the average speed $\overline{v_{s}}$ of all vehicles currently travelling along that 
Fig. 2. Topology of the Manhattan grid.

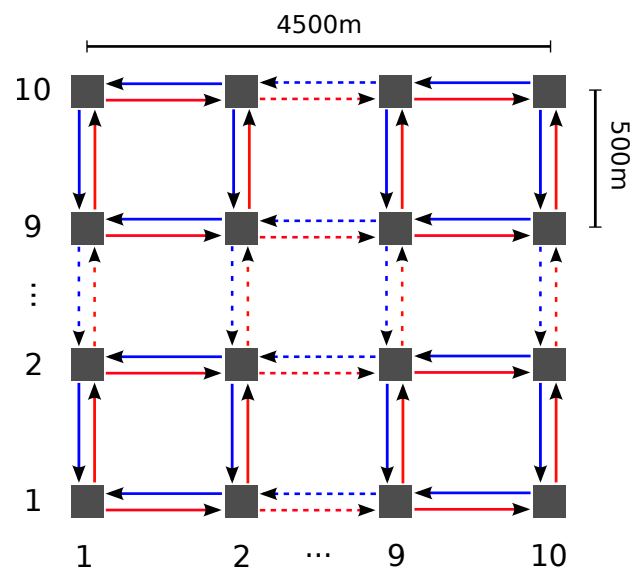

segment.

$$
\begin{gathered}
\overline{v_{s}}=\frac{1}{n_{s}} \sum_{i=1}^{n_{s}} v_{i}, \\
E T T_{s}=\frac{l_{s}}{\overline{v_{s}}} .
\end{gathered}
$$

where $n_{s}$ is the number of vehicles on the segment and $v_{i}$ is the velocity of the vehicle $i$. A route is composed of multiple segments and its overall cost $E T T_{r}$ is obtained as follows:

$$
E T T_{r}=\sum_{i=1}^{m_{r}} E T T_{s_{i}} .
$$

where $m_{r}$ is the number of segments composing the route. The minimum delay route $m d r$ between the origin $o$ and the destination $d$ is the one that minimises the ETT.

$$
m d r_{o, d}^{i}=\min \left(E T T_{R_{o, d}}\right) .
$$

The $m d r_{o, d}^{i}$ is the route provided to the vehicle $i$ from the system.

\section{EVALUATION}

In this section we evaluate the performance of our proposal for different scenarios and parameters using a microscopic traffic simulator.

Experimental setup: We used SUMO (Simulation of Urban MObility) [19], a microscopic traffic simulator and TraCI [20] as the interface to interact with the simulation at run time. As shown in Figure 2, the topology we used for all the scenarios is a 10x10 Manhattan grid with a segment length of $500 \mathrm{~m}$, in which every segment has the same priority. Every intersection follows the right-before-left priority rule and each road segment has one lane in each direction. For all our simulations we use the Krauss car-following model [14] and the vehicles were parametrized as follows:

\begin{tabular}{|l|c|}
\hline Acceleration $\left[\mathrm{m} / \mathrm{s}^{2}\right]$ & 0.8 \\
Deceleration $\left[\mathrm{m} / \mathrm{s}^{2}\right]$ & 4.5 \\
Sigma & 0.5 \\
Length $[\mathrm{m}]$ & 5 \\
Max Speed $[\mathrm{m} / \mathrm{s}]$ & 70 \\
\hline
\end{tabular}

where Sigma is the driver imperfection (defined between 0 and 1) [14]. Each trip has a source and destination chosen at random among all the edges in the Manhattan topology. The route for each trip is computed using duarouter [19], a tool provided by SUMO that uses Dijkstra's algorithm to choose the path having the minimum cost. Here the cost is determined by the length and priority of the segment.

We used two different simulation set-ups. The first has a fixed duration of one hour with a constant traffic density, i.e. additional vehicles are being inserted in the simulation when needed. With this set-up the parameters explored are the average density of vehicles in the whole scenario, the participation rate of vehicles in the experiment and the monitoring interval. In the second set-up the number of vehicles is fixed and the simulation stops when every vehicle has reached its destination. With this set-up we measured the time necessary to serve the complete traffic demand, the average velocity, the waiting time and the traffic flow distribution. In every experiment, the comparison was made between (1) the uncoordinated traffic scenario in which the vehicles follow the route initially provided (e.g. offline navigation system) and (2) the coordinated traffic scenario in which a proportion of the vehicles is dynamically rerouted to follow the route that minimises the delay. In the first simulation set-up we used the number of vehicles that reached their destination after one hour of simulation to compare the coordinated and uncoordinated traffic scenarios.

Definitions: We define as Total Vehicles Arrived (TVA), the number of vehicles that have reached their destination at the end of the simulation. The Shortest Route is defined in terms of route length and the Minimum Delay Route in term of travel delay. The Monitoring Interval is the amount of time between two computations of the minimum delay routes for the vehicles and the possible rerouteing of vehicles if needed. The Participation Rate is the percentage of vehicles that is taking active part in the experiment, or more precisely, the vehicles that are following the suggested route.

Remark It is reasonable to assume that a vehicle that travels from one place to another will use the shortest route. In a sparse traffic situation, the shortest route and the minimum delay route will coincide. In the case of traffic congestion, the difference between the two routes can become significant for the optimisation problem. Following this logic, when the congested traffic flows are distributed all over the road network, the difference between the shortest route and the minimum delay route again becomes less significant.

Average traffic density: The traffic conditions in a metropolitan area heavily varies during the day. We decided to investigate the impact of the different average densities of vehicles on the TVA for one hour of simulation. We chose different densities that allow us to go from a sparse to a fully-congested traffic scenario. The system proposed in this paper is designed to accommodate congested and heavilycongested traffic conditions. Determining the traffic situation in real time is not an easy task [3], [4], so the system has to be effective in every traffic condition in order to be deployable. In this experiment the monitoring interval was fixed at $60 \mathrm{~s}$, the participation rate to $100 \%$ to study the upper bound performance. The different average densities taken into account ranged from 10 to 49 vehicles per $\mathrm{km}$ with an increment of 
Fig. 3. Average vehicular traffic density: improvement of TVA in the coordinated traffic scenario compared to the uncoordinated and corresponding standard deviation.

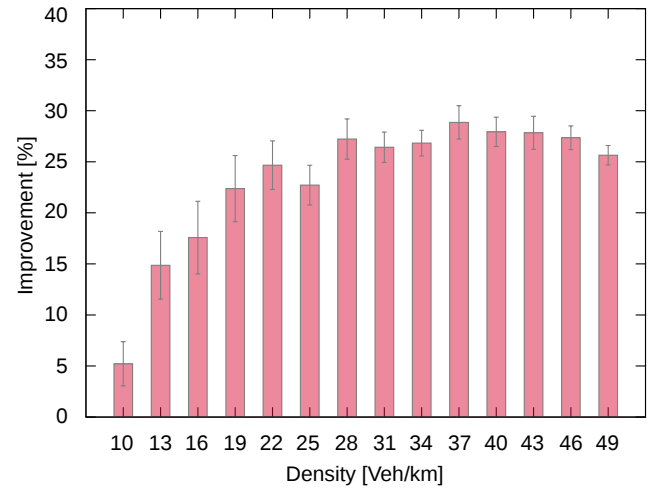

Fig. 4. Participation rate: improvement of TVA in the coordinated traffic scenario compared to the uncoordinated and corresponding standard deviation.

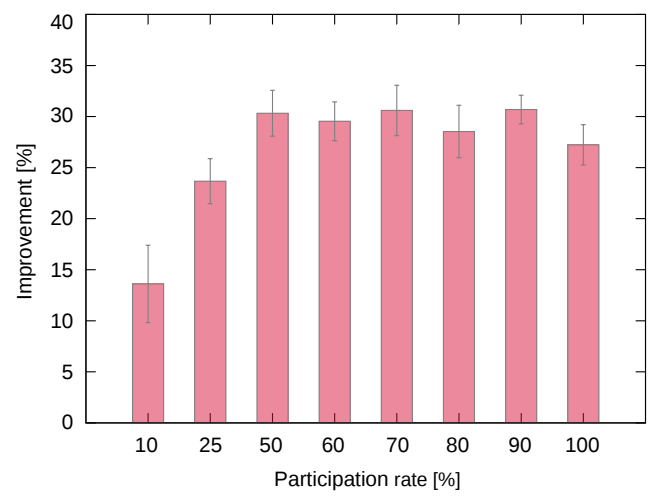

3. Figure 3 shows the percentage improvement of the TVA in the coordinated traffic scenario compared to uncoordinated one. The histogram shows, that even for a sparse traffic scenario (10), there is a TVA improvement of around 5\%. We estimated that, for our road topology, the traffic becomes heavily congested around 30 vehicles per $\mathrm{km}$; it is possible to see that the TVA keeps improving up to 28 vehicles per $\mathrm{km}$ and then, for very congested scenarios, the improvement oscillates between $24 \%$ and $29 \%$. As previously discussed, in a sparse traffic situation or with mild traffic congestion the difference between the shortest route and the minimum delay route is minimal, so the improvement for the TVA is low. On the other hand, when the system becomes heavily or fully congested, it is possible that a gridlock [21] occurs or that the road network reaches its upper bound capacity.

Participation Rate: The participation rate is critical parameter for the actual success of a system. If the protocol requires too many users to work properly, it will be hardly feasible to deploy it in a city. For this reason, we studied the impact of different participation rates on the performance of the protocol. In this case the monitoring interval is fixed to $60 \mathrm{~s}$ and the average density of vehicles per $\mathrm{km}$ to 28 . Different participation rates have been tested, ranging from $10 \%$ to $100 \%$. Figure 4 shows the percentage of improvement of the TVA in the coordinated traffic scenario compared to uncoordinated one. With $10 \%$ of active vehicles the TVA is improved by $13.3 \%$, implying that is possible to improve traffic fluidity even during the roll-out phase of the deployment (i.e.
Fig. 5. Monitoring interval: improvement of TVA in the coordinated traffic scenario compared to the uncoordinated and corresponding standard deviation.

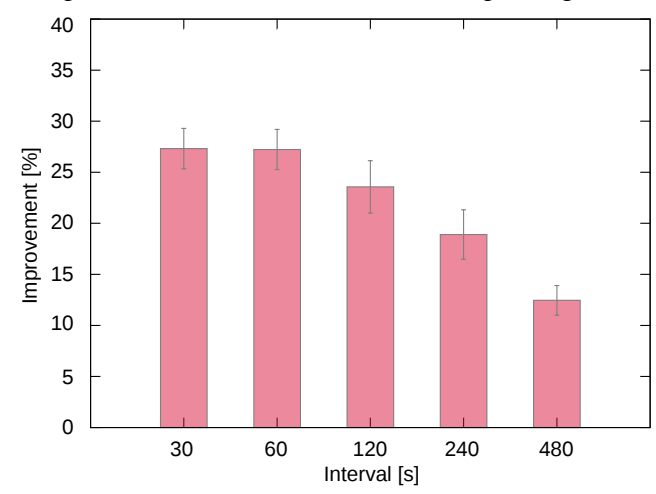

with only a few participants). By increasing the participation rate to $50 \%$, the improvement of the TVA reaches $30 \%$ and it remains stable between $27 \%$ and $30 \%$ until full participation is reached. The fluctuation of improvement with a participation rate higher than $50 \%$ is due to the randomization of the different trips for the vehicles. As mentioned previously, an average traffic density of 28 vehicles per km implies a heavilycongested scenario. This is the reason that the maximum TVA improvement is around 30\%: the traffic congestion is distributed over the whole road infrastructure and the difference between the shortest path and the minimum delay path becomes less significant. The application of the first Wardrop principle in a congested traffic situation implies that active users always experience a personal improvement by using the protocol.

Monitoring Interval: Timing plays a crucial role when it comes to the real world implementation. Even in rush hours, the global traffic situation evolves slowly and with small monitoring intervals is not possible to observe significant changes. Another factor to take into account is the amount of participating vehicles and the bandwidth consumption when the interval from one communication to the next is too short. In this experiment we wanted to investigate different monitoring intervals to find the best trade-off in terms of flow optimisation and number of transmissions. The different monitoring intervals that we used are 30,60,120, 240 and 480 seconds; for this scenario the participation rate of the vehicles is fixed to $100 \%$ and the average density of vehicles per $\mathrm{km}$ to 28 . Figure 5 shows the percentage of improvement for the TVA in the coordinated traffic scenario compared to an uncoordinated one. We can see that, with 30 and 60 second intervals, the TVA improvement is the same $(\sim 27 \%)$. This implies that is not necessary to investigate monitoring intervals shorter than 60 seconds in order to increase the performance of the system. Increasing the monitoring interval to 120 , the TVA improvement is still above 20\%; increasing the monitoring interval by repeatedly doubling the value up to 480 seconds we can see a sub-linear decrease in the TVA improvement. As the protocol does not impose strict time constraints, it will be possible to set different the monitoring intervals to optimize different aspects of the system (e.g. battery consumption in case of mobile application).

Average velocity, waiting time and traffic distribution: To compare the coordinated and the uncoordinated approach 
Fig. 6. Comparison of the duration of the simulation, the average vehicle velocity and the waiting time between the coordinated and uncoordinated traffic scenarios and corresponding standard deviation.

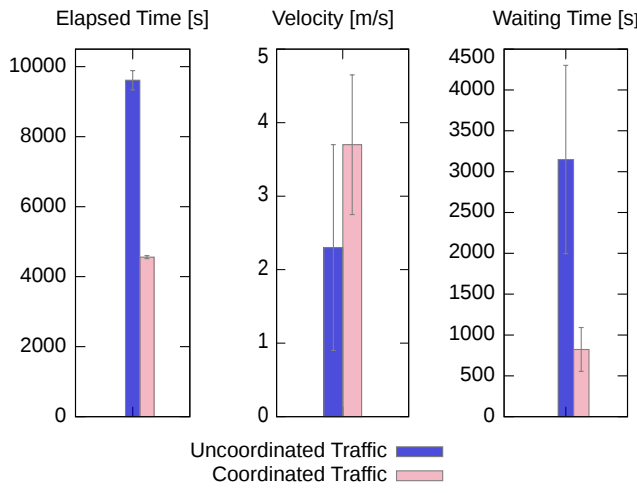

Fig. 7. Vehicular traffic density.

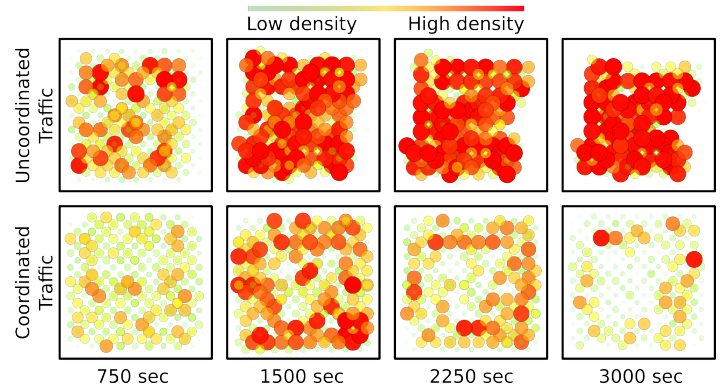

in terms of average speed and the waiting times experienced by the vehicles, we performed an additional simulation study. Using the same topology as before we fixed the participation rate to $100 \%$ and the monitoring interval to 60 s. The simulation started with 10,000 vehicles and the aim was to measure the time necessary to all of them to reach their destinations. For every vehicle, we measured the average velocity and the waiting time, defined as the amount of time during which the vehicle is stopped. Figure 6 presents the three results. For the coordinated traffic simulation, we can see that its duration is decreased around $52 \%$ relative to the uncoordinated case, meaning that the same traffic demand can be served in half of the time. Regarding the average velocity, in the coordinated traffic scenario the mean is $61 \%$ higher and the standard deviation is $58 \%$ lower compared to the uncoordinated case. The final histogram shows that the waiting time can be reduced by $74 \%$ as compared to the uncoordinated environment. These last two results have a direct impact on the possible usage and adoption of this protocol: waiting time and velocity are factors that users readily notice and with this system every vehicle would immediately experience improvements.

In the case of flow optimization, traffic distribution plays a crucial role. Figure 7 compares four snapshots of the traffic density for every road segment at different simulation times, the intervals being based on the shortest simulation (the coordinated case). In the coordinated scenario, the traffic flows are distributed over the road topology by applying the first Wardrop equilibrium. We can see that, by coordinating the behaviour of the vehicles from the outset it is possible to delay or even avoid gridlocks. This result is shown in the snapshot at simulation time 1,500 where the gridlock effect, indicated by circles with high density traffic, is present only in the uncoordinated traffic simulation.

Remarks The common result in the above experiments is the increased capacity of the system due to better usage of the road network. The two possible interpretations are that (1) the same amount of demand can be served in less time or (2) that the system is able to serve an increased demand with the same level of congestion. To give an example with numbers, in the uncoordinated traffic scenario with a density of 28 vehicles $/ \mathrm{km} / \mathrm{lane}$ in one hour of simulation the TVA is around 9,800 . In the coordinated traffic scenario with an interval of 60 seconds the corresponding TVA is around 12,300.

\section{RELATED WORK}

Over recent decades a lot of work has been done to define models, analyse traffic patterns and optimise traffic flows in metropolitan areas.

For example, NAVOPT [22] is a vehicular route optimiser that utilises traffic information gathered from vehicles to estimate travel times and to find optimal routes. It uses a Flow Deviation routing algorithm to compute optimal routes. NAVOPT improves average speed by about $25 \%$ compared to shortestpath routing and reduces total travelling time by $40 \%$.

A mathematical model is described in [21]. The authors show an adaptive control approach to relieve congestion and improve urban mobility. The basic idea consists in dividing the city into neighbourhoods of dimensions comparable to trip length and to shift the modelling emphasis from microscopic predictions to macroscopic monitoring and control. This paper proposes this idea and also discusses its relation to reality and the need for validation of the assumptions.

A later mathematical model is presented in [23], where the aim is to find a Wardrop equilibrium in transport networks in the case of uncertain situations. The authors investigate user-optimised and system-optimised transportation networks. Using Dempster-Shafer theory to find the paths with minimal cost, they modelled a game to investigate cooperative or competitive users' behaviours. This work is a theoretical counterpart to the problem we tackled in this paper.

An algorithmic solution to the closed-loop adaptive shortest path routing problem (CASPRP) is discussed in [10]. The authors solve this formulation of the problem using dynamic programming with an approximate probabilistic treatment for the labelling of the classic shortest path problem. This algorithm does not provide the full route to follow but instead indicates the best next road segment. The authors assume complete global knowledge in terms of topology of the network and current estimates of travel times on individual link. The travel time is modelled as a random variable with its mean and standard deviation predicted on the basis of a priori historical travel time information. An evaluation of the differences between the proposed algorithm and other routing algorithms is presented.

In [11] the authors evaluate different stigmergy strategies in relation to the traffic management. In their system vehicles share dynamic information and drivers can dynamically chose their route. They model the real-time information as a stigmergy. They propose anticipatory stigmergy for sharing information and to validate it with an allocation strategy which decides the 
assignment of drivers who can use the recommended route. The aim is to study the expiration time and the communication radius of the information shared among the agent to achieve better traffic management.

Another model for vehicular dynamics is that of cellular automata, used in [12] associated with a congestion-aware vehicle rerouteing strategy similar to that used in the Internet. This model is based on agents able to communicate among themselves to build local knowledge. The congestion-aware strategy is based on the level of local knowledge of the agents and their decision to chose a less-congested route.

Another system developed to optimise travel delay is Transitr [24]. The authors describe the development of a bus transit trip planner for mobile devices and evaluate its performance. They predict the shortest paths between any two points in the transit network using real-time information provided by a thirdparty bus arrival prediction system. To assess the optimality and accuracy of the prediction they make an a posteriori comparison with a schedule-based transit trip planner and the GPS traces of the transit vehicles.

To the best of our knowledge, this paper is the first to propose a deployable traffic management system based on the first Wardrop principle and evaluate it using a microscopic traffic simulator.

\section{CONCLUSIONS AND FUTURE WORK}

In this paper we presented a traffic management system to coordinate vehicular flows in urban environments. It is based on real-time traffic information gathered by an OnBoard Units and transmitted via a mobile network to the local Traffic Coordination Point (TCP) for aggregation. The routing algorithm is based on the first Wardrop principle and computes the minimum delay route using Dijkstra's algorithm with dynamic edge costs. We evaluated the system using SUMO as a microscopic traffic simulator on a 10x10 Manhattan road topology. The results show that our system is able to increase the capacity of the road network due to a better distribution of the traffic demand. Further we show that the global travel time is reduced by $50 \%$, the average speed is increased by $60 \%$ and the average waiting time is reduced by $70 \%$ compared to the uncoordinated traffic scenarios. Moreover this system reaches its full potential at participation rate of only 50\%, allowing it to be deployed in cities with an immediate gain for early adopters.

In this work we used a Manhattan topology to better control the environment and allow a precise and reliable comparison between the coordinated and uncoordinated traffic scenarios. In our future work we plan to use more complex road topologies with different type of intersections, a greater number of lanes and different priority rules. Further, we plan to investigate how the second Wardrop equilibrium can be applied to evaluate a system-wide optimisation. In this direction it will be important to find a trade-off between the system- and user-optimal equilibria. One possibility is to use incentives to persuade users to avoid selfish behavior to increase the global system performance.

\section{ACKNOWLEDGMENT}

The present project is supported by the National Research Fund, Luxembourg.

\section{REFERENCES}

[1] Wong, G. C. K. et al. (2002). A multi-class traffic flow model: an extension of LWR model with heterogeneous drivers. Transportation Research Part A: Policy and Practice.

[2] Newell, G. F. (2002). A simplified car-following theory: a lower order model. Transportation Research Part B: Methodological.

[3] Geroliminis, N. et al. (2008). Existence of urban-scale macroscopic fundamental diagrams: Some experimental findings. Transportation Research Part B: Methodological.

[4] Daganzo, C. F. et al. (2008). An analytical approximation for the macroscopic fundamental diagram of urban traffic. Transportation Research Part B: Methodological.

[5] Frank, R. et al. (2013) Energy-Efficient Rate-Adaptive Passive Traffic Sensing using Smartphones. Proceedings of the 12th Annual Mediterranean Ad Hoc Networking Workshop, MedHocNet 2013

[6] $\mathrm{Li}, \mathrm{X}$. et al. (2009). Performance evaluation of vehicle-based mobile sensor networks for traffic monitoring Vehicular Technology, IEEE Transactions on.

[7] Kjaergaard, M. B. et al. (2009). Entracked: energy-efficient robust position tracking for mobile devices in Proceedings of the 7th international conference on Mobile systems, applications, and services, ser. MobiSys 09 .

[8] Paek, J. et al. (2010). Energy-efficient rate-adaptive GPS-based positioning for smartphones in Proceedings of the 8th international conference on mobile systems, applications, and services, ser. MobiSys 10.

[9] Thiagarajan, A. et al. (2009). VTrack: accurate, energy-aware road traffic delay estimation using mobile phones. In Proceedings of the 7th ACM Conference on Embedded Networked Sensor Systems.

[10] Fu, L. (2001). An adaptive routing algorithm for in-vehicle route guidance systems with real-time information. Transportation Research Part B: Methodological.

[11] Kanamori, R. et al. (2012). Evaluation of anticipatory stigmergy strategies for traffic management. In Vehicular Networking Conference (VNC). IEEE.

[12] Scellato, S. et al. (2010). Traffic optimization in transport networks based on local routing. The European Physical Journal B.

[13] J. G. Wardrop. (1952). Some theoretical aspects of road traffic research. Proceedings of the Institute of Civil Engineers, Part II.

[14] Krauss, S. (1998). Microscopic modeling of traffic flow: Investigation of collision free vehicle dynamics. Diss. Universitat zu Koln.

[15] Nagel, K. et al. (1992). A cellular automaton model for freeway traffic. Journal de Physique I.

[16] Braess, D. et al. (2005). On a paradox of traffic planning. Transportation science.

[17] Correa, J. R. et al. (2010). Wardrop Equilibria. Wiley Encyclopedia of Operations Research and Management Science. John Wiley \& Sons, Inc.

[18] Chen, J. C. (2003). Dijkstras shortest path algorithm. Journal of Formalized Mathematics 15.

[19] Behrisch, M. et al. (2011). SUMO - Simulation of Urban MObility: An Overview In: SIMUL 2011, The Third International Conference on Advances in System Simulation.

[20] Wegener, A. et al. (2008). TraCI: an interface for coupling road traffic and network simulators. In Proceedings of the 11th communications and networking simulation symposium. ACM.

[21] Daganzo, C. F. (2007). Urban gridlock: Macroscopic modeling and mitigation approaches. Transportation Research Part B: Methodological.

[22] Kim, W. et al. (2011). NAVOPT: Navigator Assisted Vehicular route OPTimizer. In Innovative Mobile and Internet Services in Ubiquitous Computing (IMIS). IEEE.

[23] Szucs, G. et al. (2010). Finding equilibrium in transport networks by simulation and investigation of behaviors.

[24] Jariyasunant, J. et al. (2011). Mobile transit trip planning with real-time data. 